\title{
Iterative Detection of Multicode DS-CDMA Signals With Strong Nonlinear Distortion Effects
}

\author{
Rui Dinis, Member, IEEE, and Paulo Silva
}

\begin{abstract}
Whenever a direct-sequence code-division multipleaccess (DS-CDMA) signal is the sum of several components associated with different spreading codes [e.g., the DS-CDMA signal to be transmitted by the base station (BS) in the downlink or any multicode DS-CDMA signal], it has high envelope fluctuations and a high peak-to-mean envelope power ratio (PMEPR), setting strong linearity requirements for the power amplifiers. For this reason, it is desirable to reduce the envelope fluctuations of the transmitted signals. The use of clipping techniques combined with frequency-domain filtering was shown to be an effective way of reducing the envelope fluctuations (and, inherently, the PMEPR) of DS-CDMA signals, while maintaining the spectral occupation of the corresponding conventional DS-CDMA signals. To avoid PMEPR regrowth effects, the clipping and filtering operations can be repeated several times. However, the performance degradation due to nonlinear distortion effects on the transmitted signals can be relatively high, particularly when a very low PMEPR is intended (e.g., when a low clipping level and several iterations are adopted). This can particularly be serious if different powers are assigned to different spreading codes. To avoid significant performance degradation in these situations, we consider an improved receiver where there is an iterative estimation and cancellation of nonlinear distortion effects. Our performance results show that the proposed receiver allows significant performance improvements after just a few iterations, even when we have strong nonlinear distortion effects.
\end{abstract}

Index Terms-Direct-sequence code-division multiple access (DS-CDMA), envelope fluctuations, iterative detection, nonlinear distortion, signal processing.

\section{INTRODUCTION}

D IRECT-SEQUENCE code-division multiple-access (DSCDMA) schemes have been selected for several wireless systems, particularly for their good capacities and performance in time-dispersive channels [1], [2]. These techniques are good candidates for broadband wireless systems, particularly when combined with cyclic prefix (CP)-assisted block trans-

Manuscript received September 19, 2008; revised February 26, 2009. First published April 14, 2009; current version published October 2, 2009. This work was supported in part by Fundação para a Ciência e Tecnologia under pluriannual funding, U-BOAT project PTDC/EEA-TEL/67066/2006, and the FCT/POCI 2010 under Research Grant SFRH/BD/24520/2005. The review of this paper was coordinated by Dr. T. Taniguchi.

R. Dinis is with the Instituto de Telecomunicações, 1049-001 Lisbon, Portugal, and also with the Faculdade de Ciências e Tecnologia, Universidade Nova de Lisboa, 2829-516 Caparica, Portugal (e-mail: rdinis@fct.unl.pt).

P. Silva is with the Instituto de Telecomunicações, 1049-001 Lisbon, Portugal, and also with the Instituto Superior de Engenharia, Universidade do Algarve, 8005-139 Faro, Portugal (e-mail: psilva@ualg.pt).

Color versions of one or more of the figures in this paper are available online at http://ieeexplore.ieee.org.

Digital Object Identifier 10.1109/TVT.2009.2020907 mission techniques and frequency-domain equalization (FDE) schemes [3], [4].

However, envelope fluctuations and the peak-to-mean envelope power ratio (PMEPR) of DS-CDMA signals can be very high when we combine a large number of signals with different spreading codes, namely, at the downlink transmission and/or for multicode CDMA schemes [5], leading to amplification difficulties. For this reason, several techniques were proposed for designing low-PMEPR DS-CDMA signals, e.g., by adding unused codes [6] or through suitable signal processing techniques [7]-[9].

A simple and promising method for reducing the PMEPR of DS-CDMA signals is to employ a nonlinear clipping operation in the time domain followed by a linear frequencydomain filtering operation to generate a low-PMEPR version of the DS-CDMA signal, occupying the same bandwidth of the corresponding conventional DS-CDMA signal [8], [9] (similar techniques have also been proposed for reducing the envelope fluctuations of orthogonal frequency-division multiplexing (OFDM) signals [10]). However, the filtering operation produces some envelope fluctuation regrowth, limiting the achievable PMEPR [9]. As with OFDM schemes [11], [12], by repeating the clipping and filtering $(\mathrm{C} \& \mathrm{~F})$ procedures, we can reduce the PMEPR regrowth in multicode DS-CDMA schemes [13]. However, nonlinear distortion levels increase when we repeat the $\mathrm{C} \& \mathrm{~F}$ procedures, leading to performance degradation. This performance degradation can particularly be high when we have different powers assigned to different spreading codes, particularly for the spreading codes with lower power [14]. A scenario where this effect might be significant is for multiresolution broadcasting systems [15], [16], where we simultaneously transmit several parallel data streams with different powers to have different error protections. For DSCDMA systems, this can be achieved by assigning to each resolution a subset of the available spreading codes and a different power to each subset [i.e., the spreading codes with higher power have higher error protection and therefore are associated to the basic (lower) resolution].

We can significantly improve the performance of nonlinearly distorted OFDM schemes by employing enhanced receivers with iterative estimation and cancellation of nonlinear distortion effects [17], [18]. This concept was extended to multicode DS-CDMA systems in [14]. To cope with time-dispersive channels, a linear FDE was employed before the iterative estimation and cancellation of nonlinear distortion effects. However, errorpropagation effects preclude an efficient estimation and cancellation of nonlinear distortion effects when in the presence of 
severe nonlinear distortion and/or at moderate and low SNRs. In fact, for those conditions, the performance with iterative estimation and cancellation of nonlinear effects can be worse than that without compensation [18]. When suitable channel coding schemes are employed, the working region usually corresponds to low or moderate SNR values, which reduces the interest of those techniques for the estimation and cancellation of nonlinear distortion effects.

Usually, the FDE is optimized under the minimum-meansquare-error (MMSE) criterion to avoid noise enhancement effects inherent to a zero-forcing-based optimization, particularly when we have deep in-band notches [19]. This means that the spreading codes are not perfectly orthogonalized by a linear FDE, which might lead to severe interference levels, particularly when different powers are assigned to different spreading codes. It is well known that nonlinear equalizers can significantly outperform linear equalizers. A promising iterative block decision feedback equalization (IB-DFE) approach was proposed for CP-assisted single-carrier schemes [20], which can be regarded as a DFE where both the feedforward and the feedback parts are implemented in the frequency domain. This concept was extended to scenarios with space diversity [21] and to layered space-time systems [22], [23]. These schemes were also shown to be very efficient for the downlink of CP-assisted CDMA systems [24], [25].

In this paper, we consider multiresolution broadcasting systems using CP-assisted DS-CDMA schemes. We have several parallel streams with different powers to achieve multiresolution. The transmitted signals have a very low PMEPR due to the adoption of clipping (with a low clipping level) and filtering techniques, which are repeated several times. We modify the approach in [14] to cope with its major limitations, namely, the use of a linear FDE, the separate implementation of the FDE part and estimation and compensation of nonlinear distortion effects, error propagation effects in the estimation and compensation of nonlinear distortion, and poor performance in the presence of severely nonlinear distortion effects and/or at low to moderate SNRs. We consider an iterative frequency-domain receiver where the FDE operation and the estimation and cancellation of nonlinear distortion effects are jointly performed in an iterative way. Therefore, our receiver can be regarded as an IB-DFE receiver with estimation and cancellation of nonlinear distortion effects for each iteration. To improve the performance at low to moderate SNRs, we consider a turbo receiver combined with a threshold-based cancellation of nonlinear distortion effects. We also consider a turbo variant of our receiver where the channel decoding is performed for each iteration. We include the statistical characterization of the transmitted signals, which can be regarded as the extension of the approach in [9] to the case where the $\mathrm{C} \& \mathrm{~F}$ operations are repeated several times. This characterization is then used to obtain the receiver parameters.

This paper is organized as follows. In Section II, we describe the transmitter structure. Section III presents a statistical characterization of the transmitted signals, which is used for performance-evaluation purposes in Section IV. Sections V-A-C describe the receiver structure proposed in this paper, and Section V-D discuses some implementation issues.
A set of performance results is presented in Section VI, and Section VII is concerned with the conclusions of this paper.

\section{TRANSMitTER STRUCTURE}

In this paper, we consider the downlink transmission in DS-CDMA systems employing CP-assisted block transmission techniques combined with FDE schemes. The base station (BS) simultaneously transmits data blocks for $N_{R}$ resolutions. For the sake of simplicity, we assume an orthogonal spreading with $K_{r}$ spreading codes associated with the $r$ th resolution and the same spreading factor $K$ for all spreading codes. This means that

$$
\sum_{r=1}^{N_{R}} K_{r} \leq K
$$

We have a separate channel coding chain for each resolution (channel encoder, interleaver, etc.), as shown in Fig. 1(a). The coded bits associated to the $r$ th resolution are interleaved and mapped in the symbols $\left\{a_{m, r^{\prime}} ; r^{\prime} \in \Psi_{r}\right\}$, with $\Psi_{r}$ denoting a set with the indices $r^{\prime}$ of the spreading codes associated with the $r$ th resolution (naturally, it is assumed that $\Psi_{r_{1}} \cap \Psi_{r_{2}}=\emptyset$ for $r_{1} \neq r_{2}$, i.e., different spreading codes are assigned to different resolutions). For the sake of simplicity, we assume that all $K$ orthogonal spreading codes are used ${ }^{1}$ (the extension to other cases is straightforward), which means that

$$
\bigcup_{r=1}^{N_{R}} \Psi_{r}=\{0,1, \ldots, K-1\} .
$$

The block of chips to be transmitted by the BS is $\left\{s_{n} ; n=0,1\right.$, $\ldots, N-1\}$, where the "overall" chip symbol $s_{n}$ is given by

$$
s_{n}=\sum_{r=1}^{N_{R}} \sum_{r^{\prime} \in \Psi_{r}} \xi_{r} s_{n, r^{\prime}}
$$

with

$$
s_{n, r^{\prime}}=c_{n, r^{\prime}} a_{\lfloor n / K\rfloor, r^{\prime}}
$$

denoting the $n$th chip for the $r^{\prime}$ th spreading code $(\lfloor x\rfloor$ denotes a "larger integer not higher that $x$ "), where $\left\{c_{n, r^{\prime}} ; n=\right.$ $0,1, \ldots, N-1\}$ is the corresponding spreading sequence, and $\left\{a_{m, r^{\prime}} ; m=0,1, \ldots, N / K-1\right\}$ is the block of symbols associated to the $r^{\prime}$ th spreading code. The power assigned to the $r$ th resolution is proportional to $\left|\xi_{r}\right|^{2}$.

To reduce the PMEPR of the transmitted signals, we consider the transmitter structure proposed in [26] and depicted in Fig. 1(b). The block of modified samples $\left\{s_{n}^{T x} ; n=\right.$ $0,1, \ldots, N-1\}$ is formed from the original block of samples $\left\{s_{n} ; n=0,1, \ldots, N-1\right\}$ in the following way: The original block of samples is passed to the frequency domain by an $N$-point discrete Fourier transform (DFT), leading to the block $\left\{S_{k} ; k=0,1, \ldots, N-1\right\}$. Then, $N^{\prime}-N$ zeros are added to

\footnotetext{
${ }^{1}$ This means that we have an equality in (1).
} 
(a)

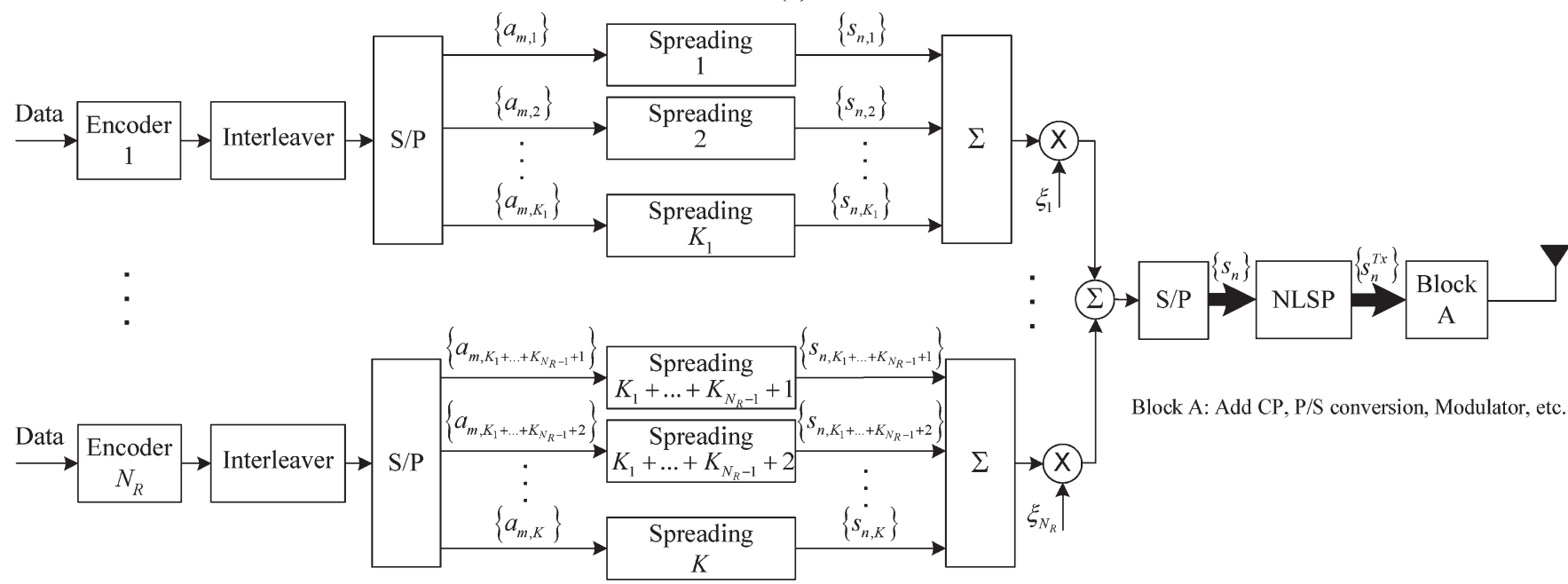

(b)

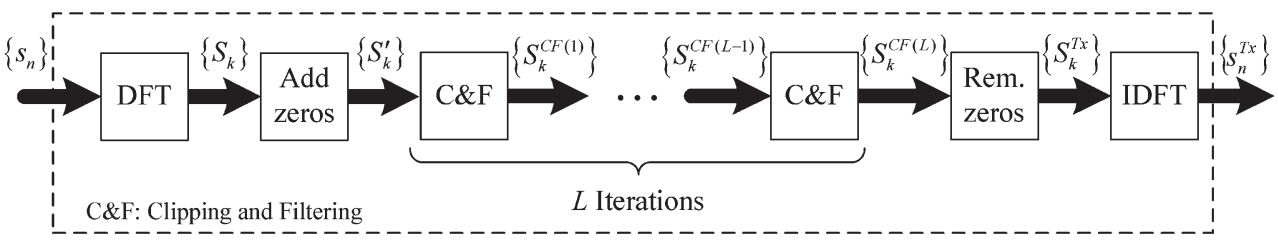

(c)

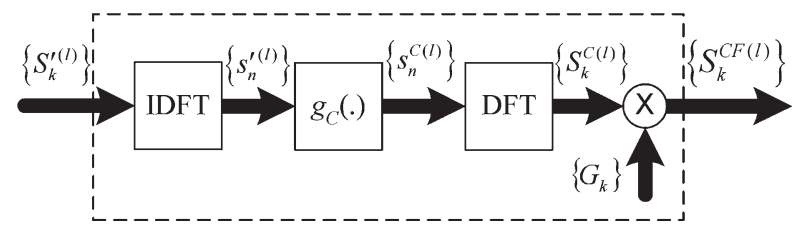

Fig. 1. (a) Transmitter structure considered in this paper. (b) Detail of the NLSP block. (c) C\&F block.

the block $\left\{S_{k} ; k=0,1 \ldots, N-1\right\}$ to form the augmented block $\left\{S_{k}^{\prime} ; k=0,1 \ldots, N^{\prime}-1\right\}$, with

$$
S_{k}^{\prime}= \begin{cases}S_{k}, & 0 \leq k<\frac{N}{2}-1 \\ S_{k-N^{\prime}}, & N^{\prime}-\frac{N}{2} \leq k<N^{\prime}-1 \\ 0, & \text { otherwise. }\end{cases}
$$

An inverse DFT (IDFT) brings the augmented block $\left\{S_{k}^{\prime} ; k=\right.$ $\left.0,1 \ldots, N^{\prime}-1\right\}$ back to the time domain [see Fig. 1(c)], resulting in the block $\left\{s_{n}^{\prime} ; n=0,1, \ldots, N^{\prime}-1\right\}$. These timedomain samples, which can be regarded as a sampled version of the DS-CDMA block, with the oversampling factor ${ }^{2} M_{T x}=$ $N^{\prime} / N$, are submitted to a nonlinear operation to reduce the corresponding PMEPR, leading to the modified samples

$$
s_{n}^{C}=g_{C}\left(\left|s_{n}^{\prime}\right|\right) \exp \left(j \arg \left(s_{n}^{\prime}\right)\right) .
$$

A possible nonlinear characteristic is an ideal envelope clipping with clipping level $s_{M}$, i.e.,

$$
g_{C}\left(\left|s_{n}^{\prime}\right|\right)= \begin{cases}\left|s_{n}^{\prime}\right|, & \left|s_{n}^{\prime}\right|<s_{M} \\ s_{M}, & \left|s_{n}^{\prime}\right| \geq s_{M} .\end{cases}
$$

${ }^{2}$ As shown in [9], $M_{T x}>1$ reduces the "in-band self-interference" effects of the nonlinearity, while increasing the "out-of-band self-interference" levels. The oversampling is also required for an effective PMEPR reduction since the envelope excursions of the samples are only similar to the excursions of the corresponding analog signal for an oversampling factor of, at least, two or four.
A DFT brings the nonlinearly modified samples back to the frequency domain, leading to the block $\left\{S_{k}^{C} ; k=0,1, \ldots, N^{\prime}-1\right\}$, where a shaping operation corresponding to a frequencydomain filtering is performed to obtain the block $\left\{S_{k}^{C F}=\right.$ $\left.S_{k}^{C} G_{k} ; k=0,1, \ldots, N^{\prime}-1\right\}$, with

$$
G_{k}= \begin{cases}1, & 0 \leq k<\frac{N}{2}-1, N^{\prime}-\frac{N}{2} \leq k<N^{\prime}-1 \\ 0, & \text { otherwise. }\end{cases}
$$

To reduce the PMEPR regrowth associated with the filtering operation, the signal processing operations that lead from $\left\{S_{k}^{\prime} ; k=0,1 \ldots, N^{\prime}-1\right\}$ to $\left\{S_{k}^{C F} ; k=0,1 \ldots, N^{\prime}-1\right\}$ in Fig. 1(c) are repeated, in an iterative way, $L$ times

$$
S_{k}^{(l)}= \begin{cases}S_{k}^{\prime}, & l=1 \\ S_{k}^{C F(l-1)}, & l>1\end{cases}
$$

where each superscript $l$ concerns a given iteration. From the block $\left\{S_{k}^{C F(L)} ; k=0,1, \ldots, N^{\prime}-1\right\}$, we form the "final" frequency-domain block $\left\{S_{k}^{T x} ; k=0,1, \ldots, N-1\right\}$ by removing $N^{\prime}-N$ zero-valued frequency-domain samples, i.e.,

$$
S_{k}^{T x}= \begin{cases}S_{k}^{C F(L)}, & 0 \leq k \leq \frac{N}{2}-1 \\ S_{N^{\prime}-N+k}^{C F(L)}, & \frac{N}{2} \leq k \leq N-1\end{cases}
$$

